\title{
Aberrant Activation Of Hedgehog Signalling Promotes Cell Migration And Invasion Via Matrix Metalloproteinase-7 In Ovarian Cancer Cells
}

\author{
Hong Zhang ${ }^{1 *}$, Yiting Wang2 ${ }^{2}$, Tingtao Chen ${ }^{3}$, Yan Zhang1, Rong $\mathrm{Xu}^{1}$, Wanwan Wang ${ }^{1}$, Minzhang Cheng2, \\ Qi Chen ${ }^{1 凶}$ \\ 1. Department of Obstetrics \& Gynecology, The Second Affiliated Hospital of Nanchang University, Nanchang, Jiangxi 330006, China \\ 2. Center for Experimental Medicine, The First Affiliated Hospital of Nanchang University, Nanchang, Jiangxi 330006, China \\ 3. Institute of Translational Medicine, Nanchang University, Nanchang, Jiangxi 330031, China \\ *Hong Zhang \& Yiting Wang contributed equally to this work \\ $\square$ Corresponding author: Dr. Qi Chen, Department of Obstetrics \& Gynecology, The Second Affiliated Hospital of Nanchang University, Nanchang, Jiangxi, \\ 330006, PR China. Email: ndefy97010@ncu.edu.cn; Fax: +86-791-88623153; Tel: +86-791-86266912. \\ () Ivyspring International Publisher. This is an open access article distributed under the terms of the Creative Commons Attribution (CC BY-NC) license \\ (https://creativecommons.org/licenses/by-nc/4.0/). See http://ivyspring.com/terms for full terms and conditions.
}

Received: 2018.04.04; Accepted: 2018.12.14; Published: 2019.01.29

\begin{abstract}
Background: Accumulating evidence indicates that aberrant activation of the Hedgehog $(\mathrm{Hh})$ signalling pathway by Glioma-associated oncogene (Gli) transcription factors is involved in the aggressive progression of cancers, including ovarian cancer. Whereas the molecular mechanism underlying this phenomenon remains unelucidated. Matrix metalloproteinase-7 (MMP-7) facilitates degradation of the extracellular matrix, promoting the invasion of tumour cells, and is associated with cancer progression and metastasis. In previous reports, we identified a set of genes regulated by $\mathrm{Hh}$ signalling in ovarian tumour cells among which MMP-7 was identified as a potential Hh target gene candidate. However, establishing an association between Hh signalling activation and MMP-7 expression requires further validation, and the function of this regulation remains unknown.
\end{abstract}

Methods: A cDNA microarray was utilized to identify potential downstream targets of $\mathrm{Hh}$ signalling. Quantitative reverse transcription polymerase chain reaction (qRT-PCR) was used to evaluate mRNA expression, and immunoblotting (IB) was conducted to evaluate protein expression. The invasive and migratory capabilities of tumour cells were tested with the transwell and wound healing assays, respectively. The mRNA levels of Gli2 and MMP-7 in normal ovarian tissues and cancerous tissues in various stages together with the corresponding clinical information were acquired from the indicated GEO datasets to elucidate associations between MMP-7 expression and cancer progression and prognosis. Additionally, immunohistochemistry $(\mathrm{IHC})$ was performed in multiple ovarian cancers, benign tumours and normal tissues to evaluate Gli2 and MMP-7 protein expression.

Results: MMP-7 expression was regulated by the $\mathrm{Hh}$ ligand, antagonist and downstream transcript factor Gli2, demonstrating this gene as an Hh target. MMP-7 facilitated the invasion and migration of ovarian tumour cells, indicating its key function in ovarian cancer progression. IHC analysis demonstrated abnormally increased Gli2 and MMP-7 expression levels in benign tumours and ovarian cancer tissues. Moreover, high MMP-7 levels were significantly associated with poor overall survival (OS) and poor progression-free survival (PFS) in ovarian cancer patients.

Conclusion: Aberrant activation of the Hh-Gli-MMP-7 signalling axis is essential for acceleration of the progression and metastasis of human ovarian cancer, implicating its use as a novel therapeutic target of ovarian cancer. In addition, MMP-7 can potentially serve as a prognostic marker of ovarian cancer.

Key words: Hedgehog, Gli, MMP-7, Ovarian cancer, Invasion, Migration 


\section{Background}

As a lethal gynaecological malignancy, ovarian cancer is the leading cause of cancer-related death among females worldwide [1, 2], with an incidence rate ranked third among all malignant tumours. Epithelial ovarian cancer is the major ovarian malignancy and comprises five histological subtypes: serous, mucinous, endometrioid, transitional and clear cell [3, 4]. With not only insidious early symptoms but also limited treatment strategies, approximately $60 \%$ of ovarian cancer patients present with distant metastasis upon initial diagnosis, and their overall 5-year relative survival rate is a miserable $46 \%$ due to clinically occult dissemination and metastasis [5]. Furthermore, the median survival of recurrent patients is generally between 1 and 2 years from the initial diagnosis $[6,7]$.

Ovarian cancer invasion and metastasis are complicated and sophisticated processes that involve a series of biochemical cascades. Several classes of proteins, including calcium-dependent cadherins, integrins, extracellular proteases, angiogenetic factors and vascular endothelial growth factor (VEGF) family members, are relevant to these processes; whereas the exact molecular mechanism underlying the invasion and metastasis of ovarian cancer still remains an area of active investigation. Therefore, it is urgent to identify significant molecular mediators that confer invasive and migratory potential to ovarian cancer cells as biomarkers for predicting risks of ovarian cancer progression and prognosis and to develop novel therapeutic strategies for ovarian cancer.

Emerging evidence has associated aberrant activation of the Hedgehog $(\mathrm{Hh})$ signalling pathway, a core regulatory network involved in animal development that is conserved from flies to humans [8], with ovarian cancer [9-11]. In mammals, the $\mathrm{Hh}$ signalling pathway is initiated by the binding of ligand, Sonic Hedgehog (Shh), Indian Hedgehog (Ihh) or Desert Hedgehog (Dhh), to its receptor, Patched (Ptch) [12]. Consequently, Ptch diminishes the inhibitory effects on Smoothened (Smo), which is localized into primary cilia (PC) and mediates activation of an intracellular cilium cascade. This activation leads to the nuclear translocation of the active form of Gli2 (Glioma-associated oncogene family member 2), a zinc finger transcriptional factor, and further transactivates the expression of target genes (e.g., Gli1, Ptch1) downstream of Hh signalling that regulate cell proliferation, apoptosis, invasion propensity, stemness, differentiation and fate determination [13]. Aberrant activation of the $\mathrm{Hh}$ signalling pathway is usually caused by a sustained increase in endogenous $\mathrm{Hh}$ expression (ligand-dependent) or by ligand-independent mutations of Patched, Smo and Suppressor of fused (Sufu) [14]. Constitutively, Hh signalling has been indicated in many observations to be involved in the development and progression of associated tumours [15-19]. Here, we focus on the pivotal role of the Hh signalling pathway in the invasion and migration of ovarian cancer, which we have previously investigated [10, 11, 20]. Specifically, we showed that activation of $\mathrm{Hh}$ signalling promoted cellular migration and invasion, whereas blockade of $\mathrm{Hh}$ signalling with GANT61 (Gli-ANTagonist 61), a small molecule inhibitor of both Gli1 and Gli2 [21], suppressed cellular migration and invasion in ovarian cancer cells. However, the exact mechanisms underlying these phenomena largely remain elusive. Our interest in matrix metalloproteinase-7 (MMP-7) greatly increased when we assessed the effects of GANT61 on the potential downstream target genes of Gli that function specifically in the cellular migration and invasion of ovarian cancer using a cDNA microarray approach; all data are available in the Gene Expression Omnibus (GEO) database under accession no. GSE53464.

Matrix metalloproteinases (MMPs), also called matrixins, were first identified by Gross and Lapiere approximately 50 years ago [22] and have been shown to facilitate cell invasion and metastasis via the enzymatic degradation of extracellular matrix (ECM) components [23]. MMP-7 is an MMP family member that comprises structural-related zinc-dependent endopeptidases [24]. This secreted protease breaks down proteoglycans, fibronectin, elastin and casein and differs from most MMP family members in that it lacks a conserved C-terminal haemopexin domain. Physiologically, MMP-7 can promote cancer invasion and angiogenesis by the proteolytic cleavage of ECM and basement membrane proteins and thus plays a vital role in the invasion and metastasis of ovarian cancer [25-27]. However, it is still among the less understood that how the expression of MMP-7 is regulated in the development and progression of ovarian cancer.

Since ECM degradation mediated by MMPs is an essential process in cancer invasion and metastasis, we hypothesize that the $\mathrm{Hh}$ pathway promotes ovarian cancer cell migration and invasion by regulating MMP-7 expression. In our current study, human ovarian cancer SK-OV-3 cells, which exhibit highly invasive behaviour, were employed to demonstrate for the first time that MMP-7 is a downstream target gene of Gli2, and aberrant activation of the canonical Hh signalling pathway promotes ovarian cell invasion and migration by regulating MMP-7. When MMP-7 was knocked down, the migration and invasion abilities of ovarian cancer 
cells were markedly decreased. Additionally, our data indicated that MMP-7 could serve as an important clinical biomarker for predicting ovarian cancer prognosis.

\section{Methods}

\section{Small molecular reagents and antibodies}

GANT61 (G9048), cyclopamine, protease inhibitor cocktail, and Lubrol-PX were purchased from Sigma-Aldrich (St. Louis, MO, USA). DMSO was purchased from Amresco and was used as the solvent for some reagents and the vehicle control. Puromycin was purchased from Salarbio. Doxycycline was purchased from Sangon Biotech (Shanghai, China). The primary antibody against Gli1 (2643S) was purchased from Cell Signaling (CST); the primary antibody against Kif3a (ab11259) were purchased from Abcam. The primary antibody against MMP-7 (C0273) was purchased from ANBO; antibody against Gli2 (sc28674) was purchased from Santa Cruz; antibody against GAPDH (MAB374) was purchased from Millipore. Enhanced chemiluminescence (ECL) Western blot detection reagents were purchased from Thermo Fisher Scientific Inc. The BLOCK-iT Pol II miR RNAi Expression Vector Kit (K4936-00) and Lipofectamine 2000 (11668-019) were purchased from Invitrogen.

\section{Cell lines and culture}

HEK293T were purchased from the American Type Culture Collection (ATCC, Manassas, VA) between 2010 and 2015. Ovarian tumour cells SK-OV-3 and ES-2 were purchased from the Cell Bank of the Chinese Academy of Sciences (Shanghai, China) between 2010 and 2013. SK-OV-3, ES-2 and 293T cells were authenticated using short tandem repeat profiling and were negative for mycoplasma contamination detecting via PCR-based assay in December 2017. The used cells were immediately expanded and frozen so that they could be resuscitated every 3 to 4 months from a frozen vial of the same batch of cells. HEK293T cells were cultured in basal Dulbecco's Modified Eagle's Medium (DMEM) supplemented with $10 \%$ foetal bovine serum (FBS, Gibco-Life Technologies) and 1\% penicillin/streptomycin, while ovarian tumour cells were cultured in McCOY's 5A medium (Gibco, 16600-082, adding NaHCO3 2.2g/L) supplemented with $10 \% \mathrm{FBS}$ and $1 \%$ penicillin/streptomycin at $37^{\circ} \mathrm{C}$ in a humidified 5\% CO2 atmosphere. In all experiments, the medium was replaced daily. Transient cell transfection was performed with Lipofectamine 2000 according to the manufacturer's instructions.

\section{Expression plasmids and N-Shh conditional medium}

The human full-length MMP-7 cDNA (GenBank accession no. NM_002423.4) construct was subcloned into pcDNA3-Flag/AB (Invitrogen). The miRNAiMMP-7 expression vectors were generated using the BLOCK-iTTM Pol II miR RNAi Expression Vector Kit (K4936-00, Invitrogen) [28, 29]. Briefly, using the human MMP-7 sequence from the NCBI gene bank, we designed three pairs of interference fragments using an Invitrogen online programme. These three regions were cloned into the pcDNA ${ }^{\mathrm{TM}} 6.2-\mathrm{GW} /$ EmGFP-miR expression vectors to construct miRNAi-MMP-7. The oligonucleotide sequences for the miRNAi constructs are listed in Additional file 1: Table S1. An expression plasmid encoding the secretory Flag-tagged N-terminal 'Hedge' domain (N-Shh), the secreted segment of the Shh ligand with ligand activity, was constructed as previously described by inserting the amino-terminal signalling domain of a human Shh cDNA (aa 26-184, GenBank accession no. NM_000193.2) into a pFlag-CMV1 vector (Sigma-Aldrich, E7273) [20, 30]. In a 10-cm dish, HEK293T cells were transiently transfected with $10 \mu \mathrm{g}$ of the Flag-N-Shh plasmid, and the empty vector plasmid was transfected into control cells. Twelve hours after transfection, the medium was replaced with basal DMEM supplemented with $2 \%$ FBS. The cells were incubated for an additional $24 \mathrm{hr}$, and the conditional medium with secreted N-Shh was then harvested and stored at $4^{\circ} \mathrm{C}$ [31]. When culturing ovarian tumour cells, the conditioned Shh medium or the control medium was mixed with an equivalent volume of fresh culture medium supplemented with $5 \%$ FBS.

\section{Lentivirus infection}

A Lenti-X-shRNA Tet-On system (pGV307-RFP) comprising shRNA-Gli2 was constructed, packaged, and purified by GeneChem (Shanghai, China), with shRNA-Gli2 targeting the sequence 5'-TCCTGAA CATGATGACCTA-3'. Lentivirus infection was performed according to the manufacturer's protocol. Briefly, stably infected cells were selected using puromycin $(1 \mu \mathrm{g} / \mathrm{ml}$, Sangon Biotech) for 7 days and induced with doxycycline $(1 \mu \mathrm{g} / \mathrm{ml}$, Sangon Biotech). Knockdown efficiency was determined by quantitative reverse transcription polymerase chain reaction (RT-PCR) and Western blot analysis.

\section{Cell migration and invasion assays}

A scratch (wounding healing) assay was used to monitor cell migration [32]. Briefly, SK-OV-3 cells were plated in 6-well plates to create a confluent monolayer and transfected with the miR-control or 
miR-MMP-7-158 plasmid at approximately 70\% confluence. Next, the cell monolayer was scraped in a straight line to create a "scratch trace" with a $200 \mu \mathrm{L}$ pipet tip. After washing the cells three times with the growth medium containing $2 \%$ FBS, the cells were treated with an equal volume of $\mathrm{N}-\mathrm{Shh}$ conditional medium or control vehicle medium for $0,12,24,36$, and $48 \mathrm{hrs}$. The cells were photographed with a phase-contrast microscope ( $10 \times$ objective lens) at 0,12 , 24,36 and 48 hrs. Wound areas were quantified using NIH Image-Pro Plus software. Data are presented as the means of three independent experiments \pm standard deviation.

Cell invasion assays were conducted in Transwell plates $(8-\mu \mathrm{m}$ pore size, $6.5-\mathrm{mm}$ diameter; Corning Life Sciences) precoated with Matrigel Basement Membrane Matrix (1 $\mathrm{mg} / \mathrm{ml}$; BD Biosciences) according to the manufacturer's protocol. First, SK-OV-3 cells were plated in 6-well plates to create a confluent monolayer and then transfected with the miR-control or miR-MMP-7-158 plasmid. After transfection, SK-OV-3 cells $\left(5.0 \times 10^{4}\right)$ in $500 \mu l$ of culture medium supplemented with $2 \%$ FBS were seeded onto the porous membrane of each insert, and $500 \mu \mathrm{l}$ of N-Shh conditional medium or control vehicle medium supplemented with $2 \%$ FBS was added to each of the bottom wells in the system. Twenty-four hours later, the cells that had migrated to the other side of the membrane were fixed with $4 \%$ paraformaldehyde and stained with crystal violet for $30 \mathrm{~min}$. The number of invading cells were photographed and counted under an inverted microscope (10× objective lens). Each experiment was repeated at least three times.

\section{RNA isolation, cDNA synthesis, and quantitative real-time PCR}

Total RNA was extracted from fresh cells using TRIzol Reagent (Invitrogen, Carlsbad, CA) and analyzed by real-time PCR. Briefly, $1 \mu \mathrm{g}$ of total RNA was reverse-transcribed to cDNA using the PrimeScript RT Reagent Kit (Takara, Japan). Finally, cDNA was analyzed using the Applied Biosystems Step One Plus ${ }^{\mathrm{TM}}$ Real-Time PCR Detection System (ABI, Foster City, CA, USA) according to the manufacturer's instructions. The real-time PCR conditions were as follows: activation at $95^{\circ} \mathrm{C}$ for $30 \mathrm{~s}$ followed by 40 cycles of denaturation at $95^{\circ} \mathrm{C}$ for $5 \mathrm{~s}$, primer annealing and extension at $60^{\circ} \mathrm{C}$ for $30 \mathrm{~s}$ and a ramp back to $95^{\circ} \mathrm{C}$. The mRNA expression levels of the target genes were normalized to those of GAPDH and quantified using the delta delta CT method. Quantitative RT-PCR for each gene was carried out in triplicate using the SYBR Premix Ex Taq RT-PCR Kit (Takara, Japan), and each experiment was repeated at least three times to ensure quantitative accuracy. The real-time PCR primer sequences are listed in Additional file 2: Table S2.

\section{Western blot analysis}

Cells were harvested after treatment with a designed programme and then subjected to Western blot analysis as described previously [28, 29]. Briefly, cells were lysed in extraction buffer comprising $0.5 \%$ Lubrol-PX, $50 \mathrm{mM} \mathrm{KCl}, 2 \mathrm{mM} \mathrm{CaCl}$, 20\% glycerol, 50 $\mathrm{mM}$ Tris- $\mathrm{HCl}$, and protease and phosphatase inhibitors ( $\mathrm{pH}$ 7.4). Cell lysates were purified by centrifugation (Eppendorf, 5415R) at 12,000 rpm for 15 $\min$ at $4^{\circ} \mathrm{C}$, and total protein concentrations were determined using a standard BCA Protein Assay Kit (23225, Thermo). Ovarian tumour cell lysates $(40 \mu \mathrm{g})$ were separated on $8 \%-10 \%$ polyacrylamide gels via SDS-PAGE and then transferred to polyvinylidene difluoride (PVDF) membranes (Millipore). Subsequent immunoblotting was performed using the following primary antibodies: Gli1 (1:500), Gli2 (1:500), MMP-7 (1:500), Kif3a (1:1000) and GAPDH (1:2000). Quantification of the immunoblot bands was accomplished using scanning films containing nonsaturated signals with the Epson V700 scanner. Densitometries of the Western blot images were quantified using ImageJ software. All experiments were repeated at least three times with consistent results.

\section{Histopathological analysis}

The excised samples were fixed in a $10 \%$ neutral buffered formalin solution, embedded in paraffin, and processed for histopathological analysis. All samples were stained with haematoxylin and eosin (H\&E), and histopathological evaluations were performed by a histopathologist blinded to the pathological diagnosis.

\section{Immunohistochemistry}

Immunohistochemistry (IHC) analyses were performed as described previously [19, 33, 34]. Briefly, tissues sections were cut into $4-\mu \mathrm{m}$-thick sections and mounted onto slides. The tissues were then deparaffinized and rehydrated, and endogenous peroxidase activity was blocked with $3 \%$ hydrogen peroxide. Then, the tissues were treated with citrate buffer $(0.01 \mathrm{M}, \mathrm{pH} 6.0)$ and heated in a microwave for $30 \mathrm{~min}$. Following a standard antigen retrieval protocol, the slides were rinsed in phosphate buffered saline (PBS) and incubated with the appropriate primary antibody, anti-Gli2 (1:100) or anti-MMP-7 (1:100), overnight at $4^{\circ} \mathrm{C}$ in a humidified chamber. Detection was achieved with a Polink-2 HRP DAB Detection Kit (Zhongshan Biotechnology) following the manufacturer's protocol. IHC images were 
captured with an FSX100 microscope (Olympus), and the German semiquantitative scoring method was employed to evaluate the scores. All stained sections, including nuclei, cytoplasms and membranes, were evaluated and scored independently by two qualified pathologists with no prior knowledge of the clinicopathological outcomes of the patients. Staining intensity was scored as 0 (no staining), 1 (weak staining), 2 (moderate staining) or 3 (strong staining), and the mean percentage of positive cells was scored as $0(0 \%), 1(1 \%-24 \%), 2(25 \%-49 \%), 3(50 \%-74 \%)$ or 4 (75\%-100\%). The final immunoreactive scores were obtained for each case by multiplying the percentage and intensity scores. Protein expression levels were further analysed by classifying the final scores as negative (-): $0-2$, low positive $(+)$ : $3-5$, medium positive $(++): 6-8$, or strongly positive $(+++)$ : 9-12. Enumeration data were obtained by classification of the final scores as one of two grades, negative $(0-2)$ or positive $(+,++,+++)$.

\section{Analyses of expression levels in tumour samples and patient survival}

For Kaplan-Meier Plotter database analysis (http://kmplot.com/), Kaplan-Meier overall survival curves were generated for ovarian patients whose follow-up data were available [35]. A log-rank (Mantel-Cox) test was used to analyse survival differences between groups.

\section{Patients and clinical tissue samples}

In total, 95 patients with ovarian epithelial cell carcinoma who underwent surgical resection at the Second Affiliated Hospital of Nanchang University between January 1998 and December 2008 (age >18 years) were included in the current study. All patients were diagnosed based on the histopathological criteria, and none of these patients had received chemotherapy, radiotherapy or immunotherapy before or after surgery. In addition, 20 patients with benign ovarian cystadenoma who underwent tumour stripping or unilateral salpingo-oophorectomy and another 10 patients who underwent both hysterectomy and bilateral or unilateral oophorectomy were selected as controls for H\&E staining and IHC analysis. Clinical samples were collected after informed consent was obtained in accordance with the Ethics Committee requirements at the participating institutes and the Declaration of Helsinki. The study procedure was approved by the Ethics Committee of the Second Affiliated Hospital of Nanchang University (Nanchang, China). Detailed clinical and pathological information is summarized in Table 3.

\section{Accession numbers}

Microarray gene expression data were deposited into the GEO database under accession number GSE53464.

\section{Statistical analysis}

Data are presented as the mean \pm SD of independent experiments performed at least three times. Paired two-tailed Student's $t$-test or one-way ANOVA were used to assess the statistical significance of differences between two different groups of quantitative data. Correlation analysis of IHC scores between Gli2 and MMP-7 expression was demonstrated using Pearson's Chi-squared test with Yates' continuity correction. Correlations were defined as follows: strong $\left(\mathrm{r}^{2}>0.75\right)$, good $\left(0.4 \leq \mathrm{r}^{2} \leq\right.$ $0.75)$, and poor $\left(\mathrm{r}^{2}<0.4\right)$ [36]. Correlations between Gli2 or MMP-7 expression and clinicopathological characteristics were analysed using $X^{2}$ tests, statistical significance was analyzed using Fisher's exact test for qualitative data. $\mathrm{P}<0.05\left(^{*}\right)$ and $\mathrm{P}<0.01\left(^{* *}\right)$ indicate statistically significant differences. SPSS software version 18.0 (SPSS, Chicago) was used to perform data analyses.

\section{Results}

\section{MMP-7 expression is regulated by $\mathrm{Hh}$ signalling.}

Hh signalling plays a vital role in the initiation and/or progression of various cancers, including ovarian cancer. To further investigate the effects of aberrant Hh signalling activation on the invasion and metastasis of ovarian cancer, we previously analysed the gene expression profile of SK-OV-3 cells treated with GANT61, a Gli inhibitor, using a cDNA microarray $[9,20]$. Herein, we focused on MMP-7 among the genes putatively down-regulated by GANT61 to elucidate the essential role of MMPs in ECM turnover and cancer cell migration [23].

While this high-throughput assay approach revealed some clues, more evidence was required to identify MMP-7 as a downstream target gene of $\mathrm{Hh}$ signalling. We first investigated whether MMP-7 responded to the Shh ligand by detecting its mRNA and protein expression in SK-OV-3 cells treated with $\mathrm{N}-\mathrm{Sh}$ or control conditional media, revealing that both the mRNA (Figure 1A) and protein (Figure 1B) expression levels of MMP-7 were increased by Shh treatment in a time-dependent manner. The protein levels of MMP-7 in ES-2 cells, another ovarian tumour cell line, either treated or not treated with Shh were then evaluated, revealing consistently increased protein expression after Shh treatment in these cells (Figure 1C). Together, these results demonstrate that 
MMP-7 expression is regulated by the Hh ligand and suggest that MMP-7 is a target gene of Hh signalling.

As Hh signalling is generally reported to be overactivated in ovarian tumours, cyclopamine, a widely used Smo inhibitor, was employed to inhibit Hh signalling in SK-OV-3 and ES-2 cells. The mRNA level of MMP-7 was decreased in SK-OV-3 cells antagonized by cyclopamine (Figure 1D), and the MMP-7 protein level was also decreased in a time-dependent manner after cyclopamine treatment in SK-OV-3 and ES-2 cells (Figure 1E and 1F). These results further confirmed MMP-7 as an Hh target gene.
A

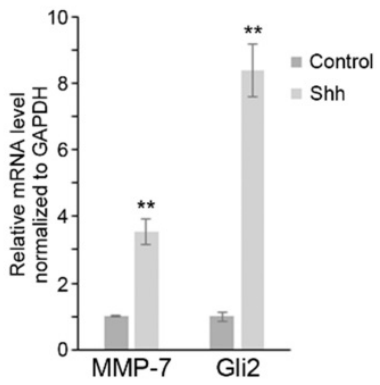

B

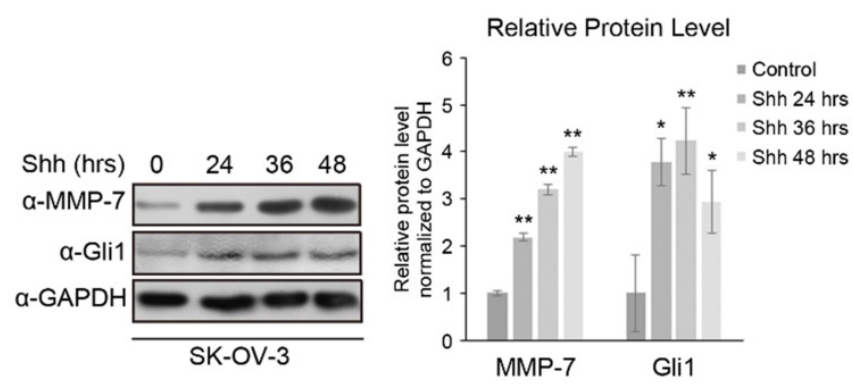

C

E

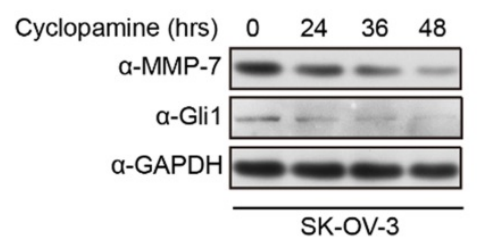

$\mathbf{F}$

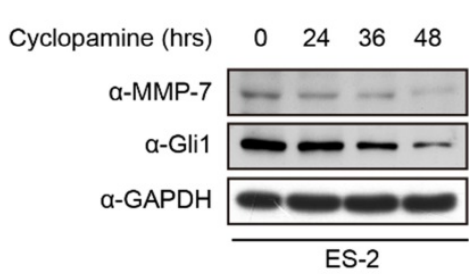

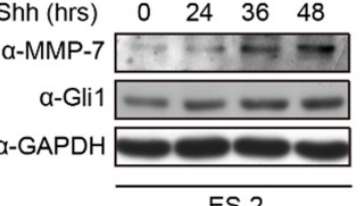

SK-OV-3

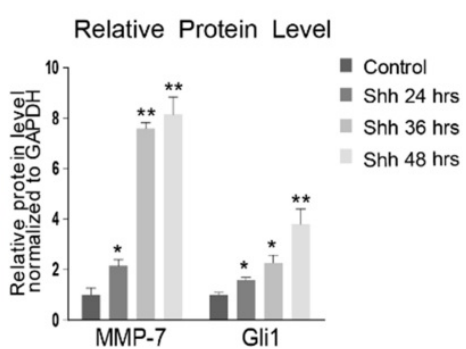

Delative mRNA Level

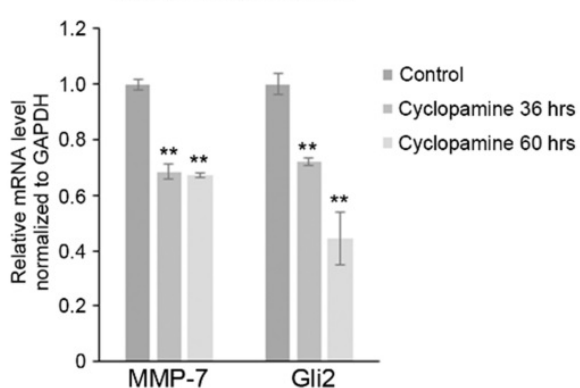

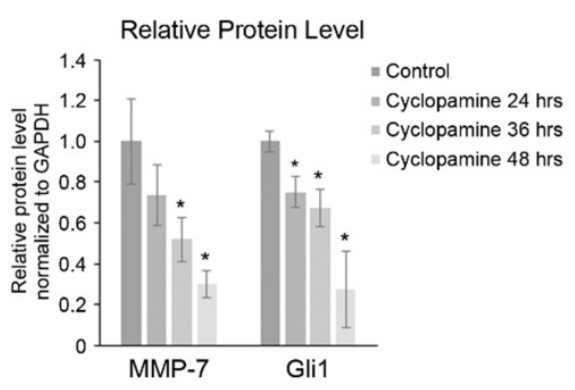

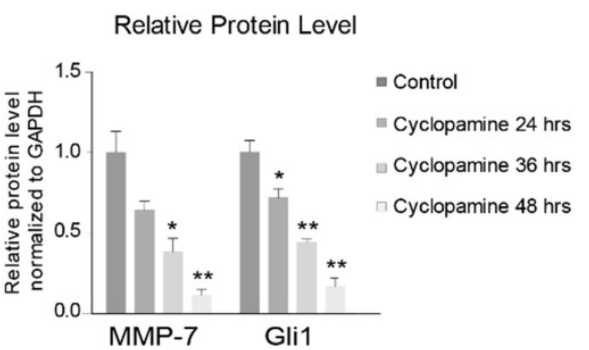

Figure 1. MMP-7 expression is regulated by the Hh signalling pathway. A. SK-OV-3 cells incubated with or without N-Shh were subjected to real-time PCR analysis for the detection of Gli2 and MMP-7 expression. Data are shown as mean \pm SD $(n=3)$. $* * P<0.01$. B. Left panel, SK-OV-3 cells incubated with N-Shh for 0 hr, 24 hrs, 36 hrs, or 48 hrs were harvested for WB analysis. Data are shown as mean \pm SD $(n=3)$. Right panel, quantification analysis of the Western blot image shown in Figure $1 B$ using Imagej software. $* p<0.05$, $* * P<0.01$. C. Left panel, ES-2 cells incubated with N-Shh for 0 hr, 24 hrs, 36 hrs, or 48 hrs were harvested for WB analysis. Data are shown as mean \pm SD ( $=3$ ). Right panel, Quantification analysis of the Western blot image shown in Figure $1 C$ using Image) software. $* P<0.05, * * P<0.01$. D. SK-OV-3 cells treated with cyclopamine $(20$ $\mu \mathrm{mol} / \mathrm{L}$ ) for $0 \mathrm{hr}, 36 \mathrm{hrs}$, or $60 \mathrm{hrs}$ were subjected to real-time PCR analysis for the detection of Gli2 and MMP-7 expression. Data are shown as mean \pm SD ( $=3$ ). **P $<0.01$. 
E. Left panel, SK-OV-3 cells treated with cyclopamine $(20 \mu \mathrm{mol} / \mathrm{L})$ for $0 \mathrm{hr}, 24 \mathrm{hrs}, 36 \mathrm{hrs}$, or 48 hrs were harvested for WB analysis with the indicated antibodies. Data are shown as mean $\pm S D(n=3)$. Right panel, Quantification analysis of the Western blot image shown in Figure $1 E$ using Image J software. $* P<0.05$. F. Left panel, ES-2 cells treated with cyclopamine $(20 \mu \mathrm{mol} / \mathrm{L})$ for $0 \mathrm{hr}, 24 \mathrm{hrs}, 36 \mathrm{hrs}$, or $48 \mathrm{hrs}$ were harvested for WB analysis with the indicated antibodies. Data are shown as mean \pm SD ( $=3)$. Right panel, quantification analysis of the Western blot image shown in Figure 1F using Image software. $* P<0.05$, $* * P<0.01$.

\section{MMP-7 expression is regulated by Hh signalling via the Gli transcription factor.}

In the canonical $\mathrm{Hh}$ signalling pathway, activated Smo inhibits the phosphorylation and ubiquitination of Glis in PC. Kinesin superfamily protein 3 (Kif3a), an essential component of the intraflagellar transport motor system (IFT) in PC, is well-known to mediate and transduce canonical $\mathrm{Hh}$ signalling. To further investigate how MMP-7 expression is regulated by $\mathrm{Hh}$ signalling, Kif3a was knocked down. Consequently, MMP-7 protein expression was reduced (Figure 2A), indicating that regulation of MMP-7 expression depends on $\mathrm{Hh}$ signalling transduction in PC.

Gli proteins shuttled from PC to the nucleus were deemed essential for transcriptional activation by Hh signalling, and serval antagonists of these proteins have been developed. SK-OV-3 and ES-2 cells were treated with GANT61 (a common Gli inhibitor) to block Hh signalling in the nucleus, which reduced MMP-7 protein expression in both SK-OV-3 and ES-2 cells in a time-dependent manner (Figure 2B and 2C). Consistent with this phenomenon, MMP-7 mRNA expression was also depressed by GANT61 treatment (Figure 2D), indicating that MMP-7 expression depends on Gli transcriptional activity.

Gli2 was knocked down in SK-OV-3 to block Gli transcriptional activity, and MMP-7 mRNA and protein expression was subsequently detected. Gli2 depletion suppressed the protein expression of MMP-7 in SK-OV-3 (Figure 2E). Additionally, the mRNA expression of MMP-7 was also decreased in SK-OV-3 cells in which Gli2 was knocked down (Figure 2F), further confirming MMP-7 as a target gene of Gli transcription factors. To further illustrate whether Gli2 or Gli1 acts the predominant role in regulating MMP-7, SK-OV-3 cells were transfected transiently with overexpression plasmids of pUB6/V5-hisB-Gli1 or empty vector, then the mRNA expression levels of Gli1 and MMP-7 were detected using $\mathrm{qPCR}$. And we found that the mRNA expression of MMP-7 did not change while Gli1 was upregulated significantly (Figure S2A). This result revealed that Gli1 did not regulate MMP-7 directly. Next, we conducted transfection of plasmids of pcMV6-entry-Gli2-myc or empty vector, then the mRNA expression levels of Gli2, Gli1 and MMP-7 were detected using qPCR. Consequently, the mRNA expression levels of MMP-7 and Gli1 were increased accordingly after overexpressing Gli2 (Figure S2B).
Therefore, our results revealed that MMP-7 is regulated directly by Gli2 other than Gli1. Taken together, these results demonstrate that MMP-7 is a target gene of Hh signalling downstream of Gli2.

\section{MMP-7 participates in the migration and invasion of ovarian tumour cells induced by $\mathrm{Hh}$ signalling.}

As modulators in ECM turnover and cancer cell migration, MMPs represent the most prominent family of proteinases associated with cancer metastasis [23]. However, the function of MMP-7 and the effects of regulating MMP-7 expression via $\mathrm{Hh}$ signalling remain unclear in ovarian tumour cells.

Three miRNAs targeting MMP-7 mRNA were designed to evaluate the endogenous function of MMP-7 in ovarian tumour cells, and their knockdown efficiencies were evaluated by detecting MMP-7 protein expression (Figure 3A) and mRNA expression (Supplementary Figure S1H). miR-MMP-7-158 and miR-MMP-7-668 dramatically interfered with the endogenous expression of MMP-7 (Figure 3A and 3B). To make a comprehensive assessment of protein and mRNA level, we choose miR-MMP-7-158 as a putative interfere construct.

Classic transwell and wound healing assays were utilized to evaluate the invasion and migration of ovarian tumour cells. Shh stimulation promoted both invasion and migration in SK-OV-3 cells, while MMP-7 depletion suppressed the invasive and migratory abilities of SK-OV-3 cells and partially ameliorated the effect of Shh treatment (Figure 3C, $3 \mathrm{D}, 3 \mathrm{E}$, and $3 \mathrm{~F}$ ). We also repeated the invasive and migratory experiments with miR-MMP-7-668 (Supplementary Figure S1D, S1E, S1F and S1G). These results indicate that as an $\mathrm{Hh}$ target gene, MMP-7 might participate in the regulation of cell invasion and migration induced by Hh signalling.

\section{MMP-7 and Gli2 expression are aberrantly increased in ovarian cancer tissues and predict poor clinical outcomes.}

MMPs reportedly play important roles in the tumourigenesis and progression of lung cancer, breast cancer, colorectal cancer, prostate cancer [18], hepatocellular carcinoma [37], and pancreatic ductal adenocarcinoma [38], among others [39]. However, whether the dysregulation of MMP-7 expression facilitates ovarian tumour progression remains unclear. 
A

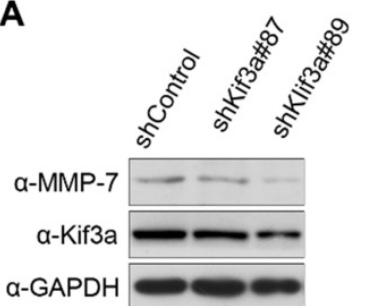

B

GANT61 (hrs) $\quad 0 \quad 24 \quad 36 \quad 48$

a-MMP-7 --

a-Gli1

a-GAPDH

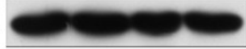

SK-OV-3

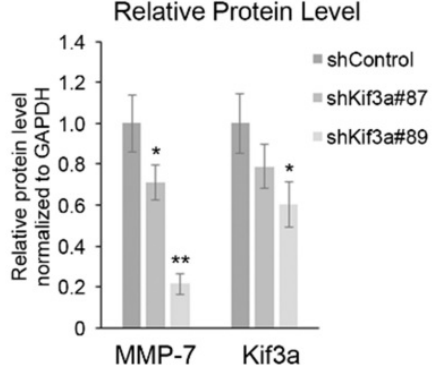

C

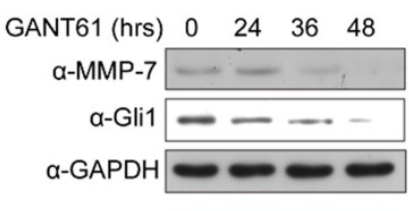

ES-2
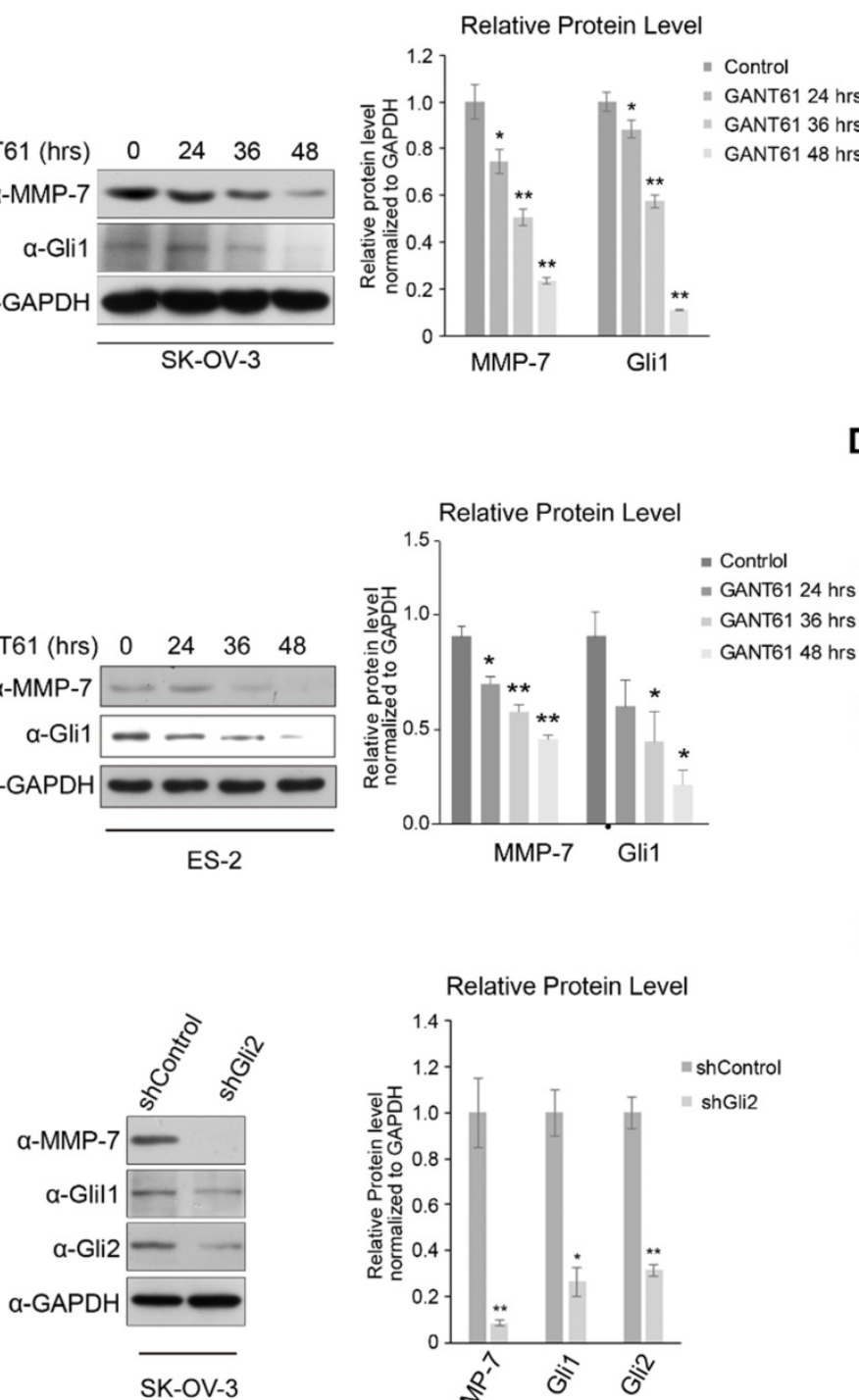
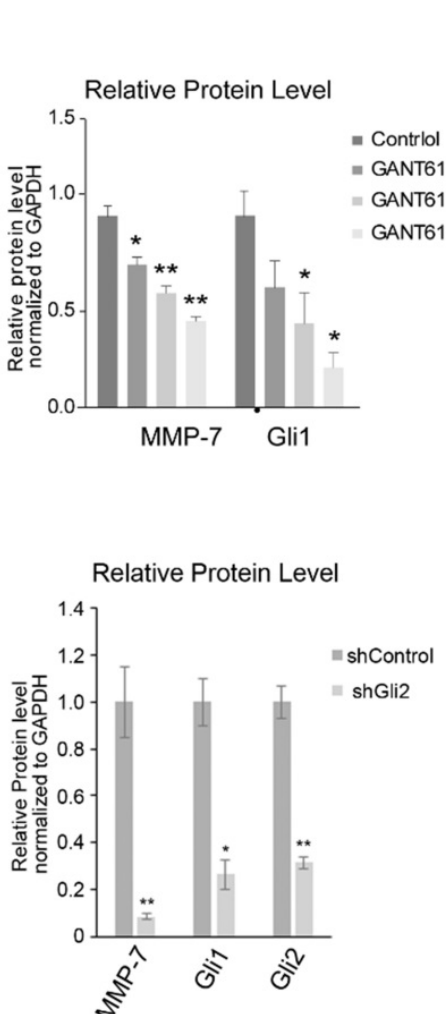

D

Relative mRNA Level

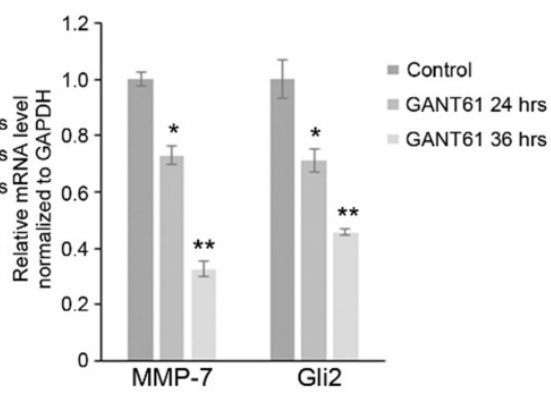

$\mathbf{F}$
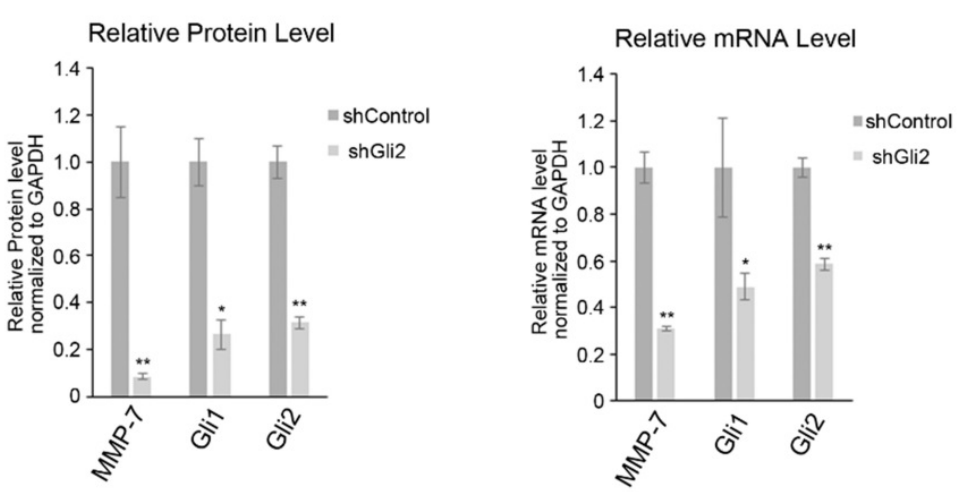

Figure 2. MMP-7 is a downstream target gene of the canonical Hh signalling pathway. A. Left panel, shRNA-Kif3a decreased the protein level of MMP-7. Right panel, quantification analysis of the Western blot image shown in Figure 2A using Imagel software. Data are shown as mean \pm SD $(n=3)$. $* P<0.05$, $* * P<0.01$. B. Left panel, SK-OV-3 cells treated with GANT61 (5 umol/L) for $0 \mathrm{hr}, 24 \mathrm{hrs}, 36 \mathrm{hrs}$, or $48 \mathrm{hrs}$ were harvested for WB analysis with the indicated antibodies. Right panel, quantification analysis of the Western blot image shown in Figure $2 B$ using ImageJ software. Data are shown as mean $\pm S D(n=3)$. $* P<0.05$, $* * P<0.01$. C. Left panel, ES-2 cells treated with GANT61 (20 $\mu \mathrm{mol} / \mathrm{L})$ for $0 \mathrm{hr}, 24 \mathrm{hrs}$, or $36 \mathrm{hrs}$, or $48 \mathrm{hrs}$ were harvested for WB analysis with the indicated antibodies. Right panel, quantification analysis of the Western blot image shown in Figure $2 C$ using Imagel software. Data are shown as mean \pm SD $(n=3)$. $* P<0.05$, $* * P<0.01$. D. ES-2 cells treated with GANT61 $(20 \mu \mathrm{mol} / \mathrm{L}) \mathrm{for} 0 \mathrm{hr}, 24 \mathrm{hrs}$, or $36 \mathrm{hrs}$ were subjected to real-time PCR analysis for the detection of Gli2 and MMP-7 expression. Data are shown as mean \pm SD $(n=3)$. $* P<0.05$, $* * P<0.01$. E. Left panel, SK-OV-3 cells were infected with lentivirus harbouring shRNAs (Lenti-shRNA-control and Lenti-shRNA-Gli2). Effective Gli2 knockdown markedly decreased the protein expression of Gli1 and MMP-7. Glil acted as a positive control. Right panel, quantification analysis of the Western blot image shown in Figure 2E using Imagej software. Data are shown as mean \pm $S D(n=3) . * P<0.05, * * P<0.01$. F. SK-OV-3 cells infected with lentivirus harbouring shRNAs (Lenti-shRNA-control and Lenti-shRNA-Gli2) were subjected to real-time PCR analysis for the detection of Gli2, Glil and MMP-7 expression. Data are shown as mean \pm SD $(n=3) * P<0.05, * * P<0.01$

MMP-7 mRNA expression in normal ovarian tissues and various types of primary ovarian tumours sourced from GEO (GSE6008) were analysed, revealing higher MMP-7 expression in certain types of 
ovarian tumours (Figure 4A). Gli2 and MMP-7 protein expression levels in normal ovarian tissues and ovarian tumour samples were evaluated by IHC analysis, revealing that both Gli2 and MMP-7 expression levels were increased in ovarian tumour samples compared with those in normal tissues (Figure 4D, and Table 1). Interestingly, the protein levels of MMP-7 showed a positive correlation with Gli2 (Figure 4D, and Table 2), suggesting that MMP-7 expression is regulated by Hh-Gli2 signalling in ovarian tumours. Moreover, high Gli2 and MMP-7 expression was significantly correlated with clinical stage and lymphatic metastasis (Table 3). These observations demonstrate that Gli2 and MMP-7 are highly expressed in ovarian cancer tissues and

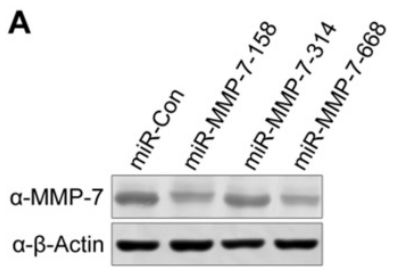

C

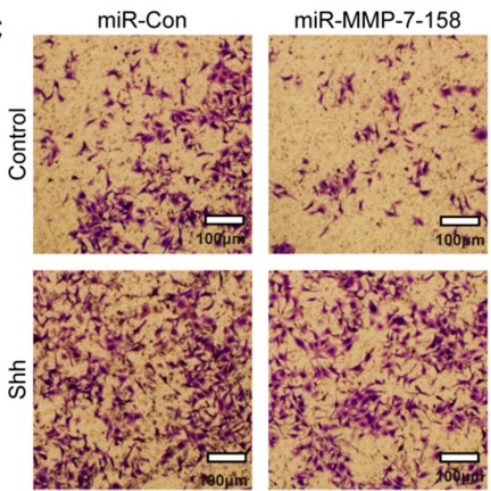

suggest their association with ovarian tumourigenesis or ovarian cancer progression.

Kaplan-Meier survival analysis of 28 ovarian cancer patients (sourced from GSE23554) stratified by varying MMP-7 expression levels revealed that high MMP-7 levels were significantly associated with poor overall survival $(p=0.0055, H R=4.38$, Figure 4B) [35]. Similarly, high MMP-7 levels in ovarian cancer patients sourced from The Cancer Genome Atlas (TCGA, n=565) also signified poor progression-free survival $(p=0.048, H R=1.3$, Figure $4 C)$ [35]. These survival correlation analyses suggest that the level of MMP-7 expression is related to poor prognosis and might be a potential marker for prognosis prediction.

B

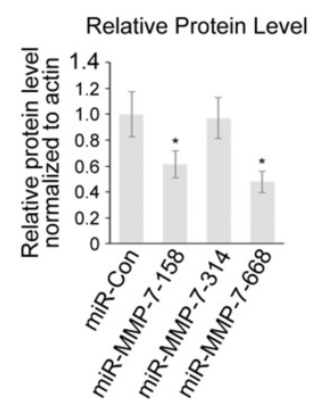

D

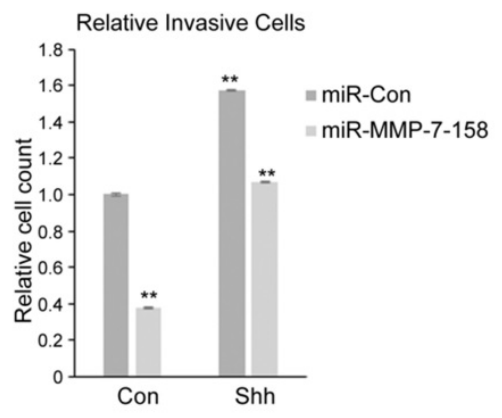

$\mathbf{F}$

E
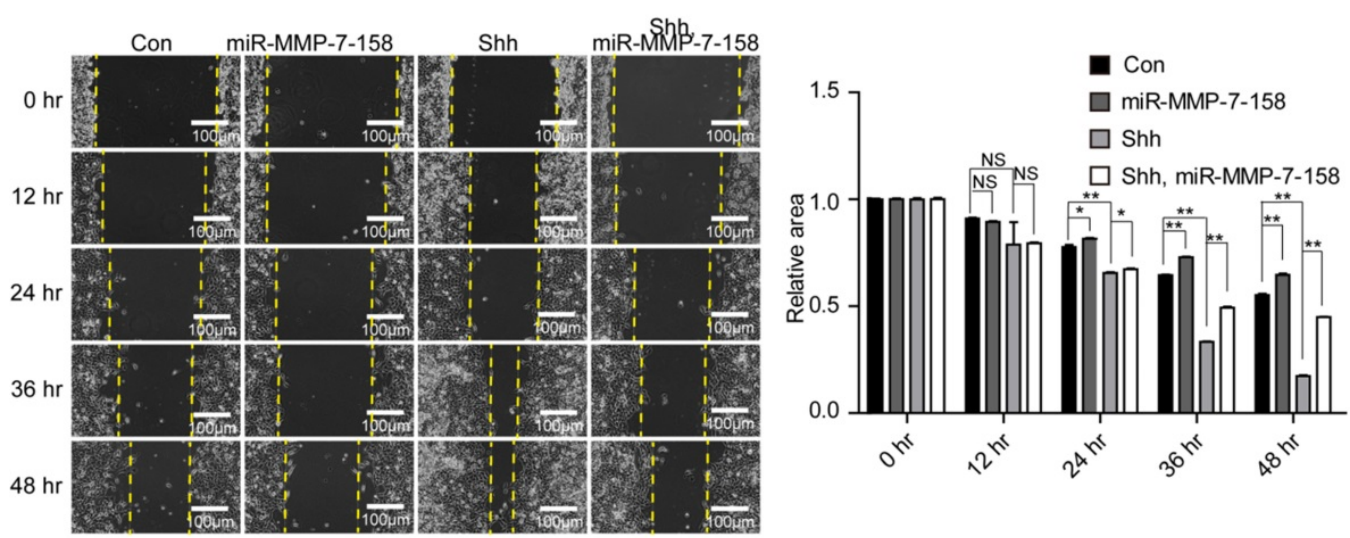

Figure 3. MMP-7 promotes the migration and invasion abilities of ovarian cancer cells. A. Validation of the knockdown efficiencies of three miR-MMP-7 constructs (miR-MMP-7-158, miR-MMP-7-314, and miR-MMP-7-668) in SK-OV-3 cells. B. Quantification analysis of the Western blot image shown in Figure 3A using Imagel software. Data are shown as mean \pm SD $(n=3) * P<0.05$. C. MMP-7 knockdown reduces N-Shh-induced invasive ability of SK-OV-3 cells. Scale bar, $100 \mu$ m. D. Quantification analysis of the cell counts shown in Figure 3C. Data are shown as mean \pm SD $(n=3)$. $* * P<0.01$. E. MMP-7 knockdown inhibits the N-Shh-stimulated migratory ability of SK-OV-3 cells. Scale bar, $100 \mu \mathrm{m}$. F. Quantification analysis of the wound areas shown in Figure 3E using NIH Image-Pro Plus software. Data are shown as mean \pm SD ( $\mathrm{n}=3$ ). $* P<0.05$, $* * P<0.01$. 
A

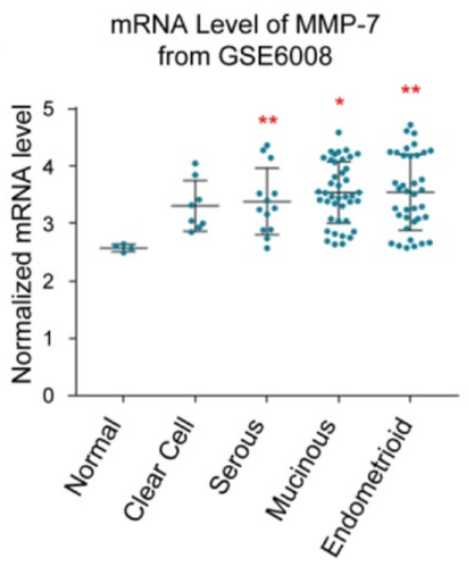

B

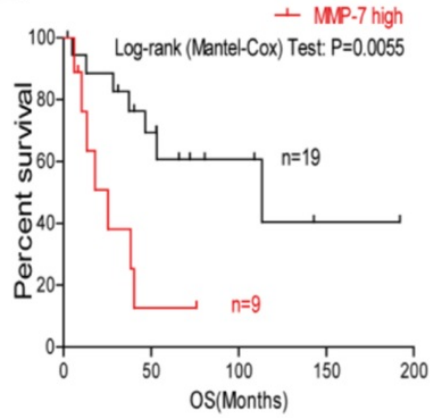

C

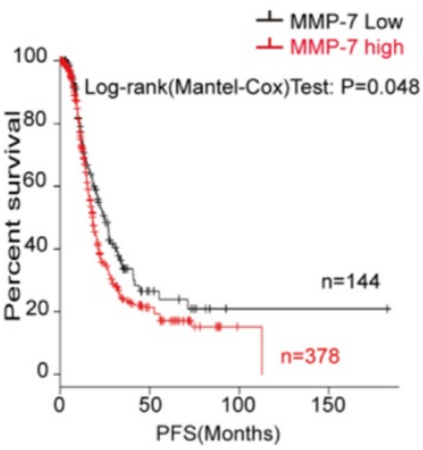

D
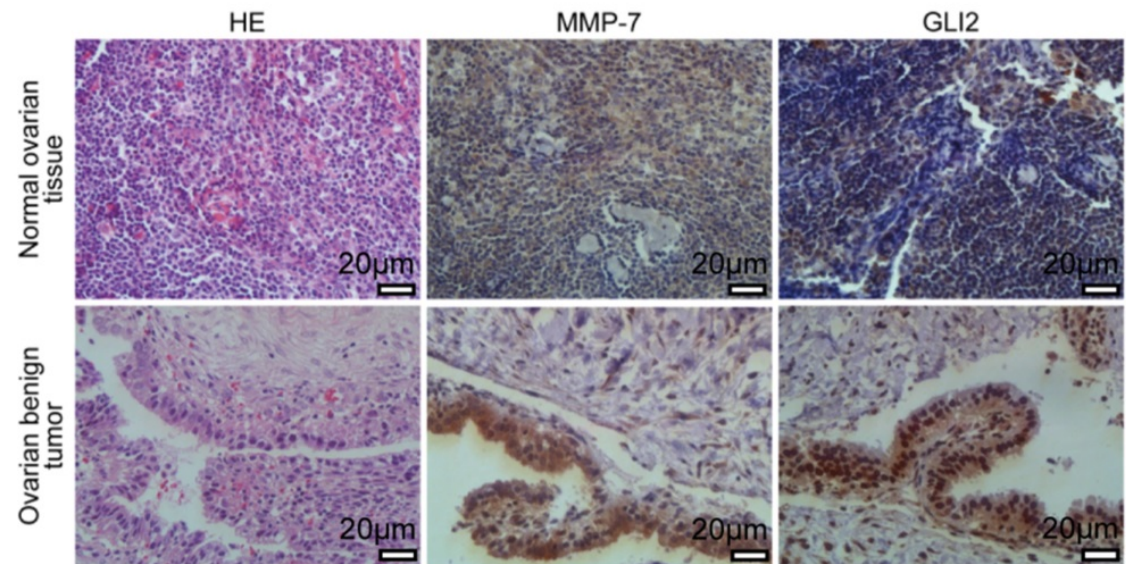

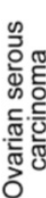
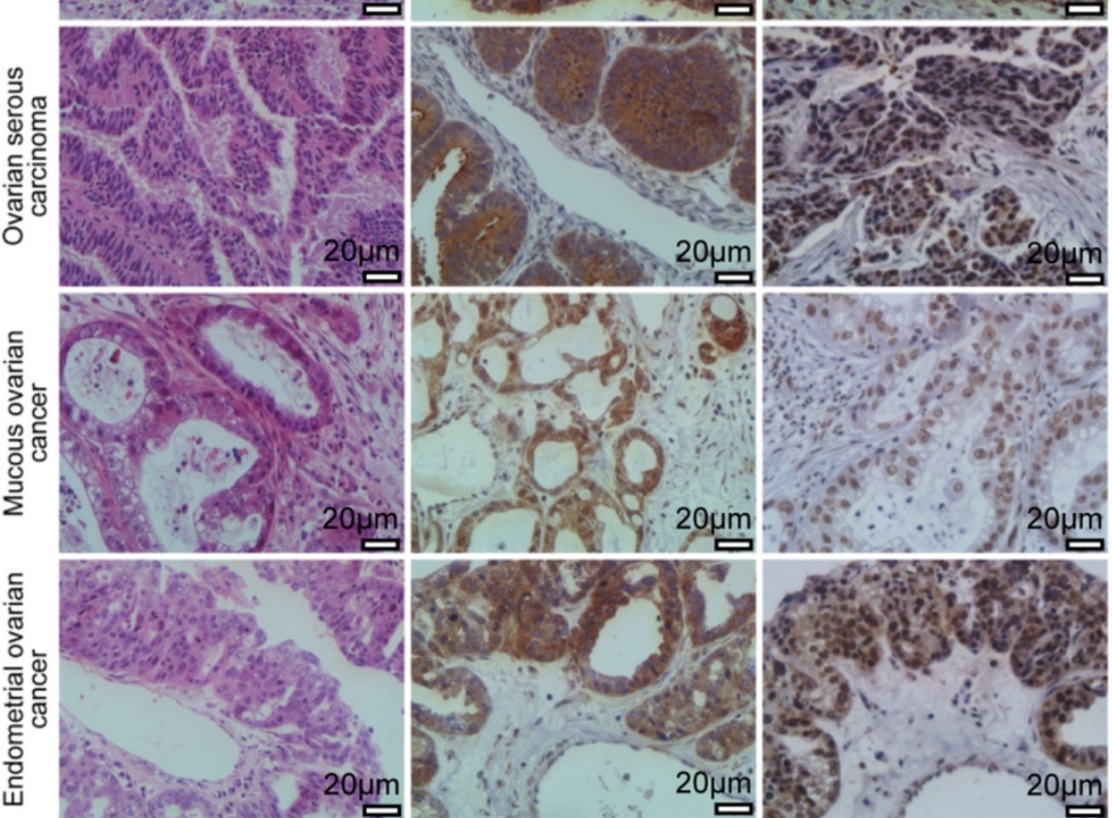

Figure 4. MMP-7 expression is abnormally elevated in ovarian cancer tissues and predicts poor clinical prognosis. A. Analysis of MMP-7 mRNA levels in the "GSE6008" dataset obtained from the publicly available Oncomine database. B. Kaplan-Meier survival rate analysis show that high MMP-7 expression is associated with poor overall survival. Overall survival data were obtained from the publicly available NCBI GEO database \#GSEGSE23554. C. Kaplan-Meier survival rate analysis show that high MMP-7 expression is associated with poor progression survival. PFS data were obtained from the publicly available TCGA database. D. Representative immunostaining of Gli2 and MMP-7 in normal tissues and benign tumour, serous carcinoma, mucous cancer, and endometrial cancer tissues. Scale bar, $20 \mu \mathrm{m}$. 
loss-of-function and dominant-negative mutations in Gli2 lead to holoprosencephaly-like features and pituitary anomalies.

Invasion and metastasis are complicated, multi-step processes in which cancer cells undergo detachment from the primary site, intravasation into blood vessels, extravasation to a different bodily location, and colonization at the secondary site [48]. Degradation of the ECM and basement membrane is a significant proteolytic event in cancer invasion and metastasis initiation [49]. MMPs, whose general architectural features demonstrate three domains, the catalytic domain, the pro-peptide domain, and the haemopexin-like C-terminal domain linked to the catalytic domain via a flexible hinge region, have traditionally been found to play vital roles in late-stage tumour cell invasion and metastasis based on their ability to degrade ECM components. Furthermore, MMPs regulate other signalling pathways that control cell growth, inflammation, and angiogenesis and may even function in a nonproteolytic manner. MMPs promote the epithelial-to-mesenchymal transition (EMT) by cleaving the cell adhesion molecule E-cadherin and liberating TGF- $\beta$. Moreover, MMPs reportedly trigger the angiogenic switch, and release VEGF during carcinogenesis [50]. Sawada revealed that ovarian cancer metastasis is most likely induced by upregulation of the alpha 5-integrin mediated by E-cadherin loss [51]. Sakata indicated that MMP-2 and MMP-9 are frequently overexpressed in ovarian cancer cells disseminated in the peritoneal cavity [52]. Laminin is an ECM component that is associated with tumour cell metastasis, and one study indicated that laminin expression in the ascitic fluid of patients with ovarian cancer is greatly increased [53]. In recent years, VEGF family members, especially placental growth factor (PLGF), have been shown to activate MMP-7-mediated ovarian cancer invasion [54].

MMP-7, a secreted proteolytic enzyme, differs from most MMP family members in that it lacks a conserved C-terminal haemopexin domain. MMP-7 exhibits elevated expression levels in multiple human cancers and has been previously demonstrated to facilitate tumour invasion, which is consistent with the results of this study. Specifically, ectopic Gli2 overexpression using the Shh ligand, an Hh signalling pathway activator, facilitated ovarian cancer cell invasion and migration (Figure $3 \mathrm{C}$ and $3 \mathrm{E}$ ). However, simultaneously inhibiting MMP-7 with an miRNA and the Shh ligand inhibited ovarian cancer cell invasion and migration. The exact mechanisms underlying the invasion and migration of ovarian cancer cells are complex. Some studies suggest that these processes are partially due to the induction of progelatinase activation [26], while another study indicates that mesothelin may participate in the invasion of ovarian cancer by inducing MMP-7 expression via the MAPK/ERK and JNK pathways [25]. Increasing evidence indicates that ligand-dependent Hh signalling, also termed canonical Hh signalling, plays an essential role in cancer. On the other hand, noncanonical mechanisms independent of $\mathrm{Hh}$ signalling also play regulatory roles in the expression of Gli target genes. This Hh-independent regulation of Gli indicates that a cross talk between the $\mathrm{Hh}$ pathway and other signalling pathways may exist during animal development and tumourigenesis. Many studies have shown an apparent cross talk between the $\mathrm{Hh}$ and Wnt pathways in ovarian cancer [55, 56]. The PI3K-Akt and Shh-Gli1 pathways are also validated in the occurrence of EMT in ovarian cancer cells [57].

High Gli1 expression in advanced serous ovarian cancers is associated with unfavourable overall survival [58]. Consistent with these findings, we found that Gli2 and MMP-7 were both markedly elevated in malignant ovarian cancer tissues (Figure 4A and 4C). Furthermore, our analysis of clinical samples also indicated that MMP-7 expression was positively correlated with Gli2. In addition, the expression of MMP-7 and Gli2 was significantly correlated with the clinical stage and lymphatic metastasis (Table 3). Finally, our analyses of public databases showed that higher levels of Gli2 and MMP-7 were associated with unfavourable survival and poor progression-free survival, which was also found in another study [58]. Furthermore, we showed that MMP-7 can potentially serve as a marker for ovarian cancer recurrence, as previously reported [59].

Collectively, we demonstrate that aberrant activation of the Hh signalling pathway promotes ovarian cancer cell migration and invasion by regulating MMP-7, suggesting that this pathway could serve as a potential target for future ovarian cancer therapies. Furthermore, our results demonstrate the feasibility of using MMP-7 as a prognostic marker and target for therapeutic ovarian cancer interventions.

\section{Conclusions}

Herein, we validated that the Hedgehog signalling pathway promotes ovarian cancer invasion and migration via matrix metalloproteinase-7. Furthermore, Gli2 and MMP-7 are aberrantly and consistently elevated in ovarian cancer, which can predict poor clinical outcomes. These observations reveal novel biological insight into how the $\mathrm{Hh}$ pathway regulates the invasion and migration of 
ovarian cancer and highlight a preclinical rationale for using MMP-7 as a potential therapeutic target and prognostic marker in ovarian cancer.

\section{Abbreviations}

Dhh: Desert Hedgehog; FBS: Foetal bovine serum; Gli: Glioma-associated oncogene; GEO: Gene Expression Omnibus; H\&E: Haematoxylin and eosin; Hh: Hedgehog; IHC: immunohistochemistry; Ihh: Indian Hedgehog; MMP-7: Matrix metalloproteinase-7; OS: Overall survival; PBS: Phosphate-buffered saline; PC: Primary cilia; PFS: progression-free survival; Ptch1: Patched 1; qRT-PCR: quantitative real-time polymerase chain reaction; Shh: Sonic Hedgehog; Smo: Smoothened; Sufu: Suppressor of fused; TCGA: The Cancer Genome Atlas.

\section{Supplementary Material}

Supplementary figures and tables. http://www.jcancer.org/v10p0990s1.pdf

\section{Acknowledgments}

This work was supported in part by grants from the National Natural Science Fundation of China (81460392) and the Jiangxi Government (20161ACB2 0014) as well as the Graduate Innovation Foundation of Jiangxi Province (YC2017-B027).

\section{Ethics approval and consent to participate}

Patient samples were obtained in accordance with the ethics committee of the Second Affiliated Hospital of Nanchang University and the Declaration of Helsinki. The study protocol of human tissue was approved by the Institutional Review Board of the Second Affiliated Hospital of Nanchang University. (Nanchang, China).

\section{Authors' contributions}

$\mathrm{HZ}$ and YW carried out the experiments of Western Blot as well as qRT-PCR; HZ analyzed and interpreted the data; $\mathrm{YW}$ drafted the final manuscript; $Y Z$ and WW analyzed and interpreted the patient data, and contributed to the immunohistochemistry experiments; TC drafted the initial manuscript; RX performed the invasion and migration assay; $\mathrm{MC}$ performed statistical analysis as well as figure construction; QC managed the experimental design, reviewed the manuscript and provided funding support. All authors read and approved the final manuscript.

\section{Competing Interests}

The authors have declared that no competing interest exists.

\section{References}

1. Burstein H J, Krilov L, Aragon-Ching J B, et al. Clinical Cancer Advances 2017: Annual Report on Progress Against Cancer From the American Society of Clinical Oncology. J Clin Oncol. 2017; 35: 1341-1367.

2. Iorio M V, Croce C M. MicroRNA profiling in ovarian cancer. Methods Mol Biol. 2013; 1049: 187-97.

3. Scully R E. Pathology of ovarian cancer precursors. J Cell Biochem Suppl. 1995; 23: 208-18.

4. Rosen D G, Yang G, Liu G, et al. Ovarian cancer: pathology, biology, and disease models. Front Biosci (Landmark Ed). 2009; 14: 2089-102.

5. Siegel R L, Miller K D, Jemal A. Cancer statistics, 2016. CA Cancer J Clin. 2016; 66: 7-30.

6. Rauh-Hain J A, Krivak T C, Del Carmen M G, et al. Ovarian cancer screening and early detection in the general population. Rev Obstet Gynecol. 2011; 4: 15-21.

7. Lengyel E. Ovarian cancer development and metastasis. Am J Pathol. 2010; 177: 1053-64.

8. Jiang J, Hui C C. Hedgehog signaling in development and cancer. Dev Cell. 2008; 15: 801-12.

9. Zeng $\mathrm{C}$, Chen $\mathrm{T}$, Zhang $\mathrm{Y}$, et al. Hedgehog signaling pathway regulates ovarian cancer invasion and migration via adhesion molecule CD24. J Cancer. 2017; 8: 786-792.

10. Liao X, Siu M K, Au C W, et al. Aberrant activation of hedgehog signaling pathway in ovarian cancers: effect on prognosis, cell invasion and differentiation. Carcinogenesis. 2009; 30: 131-40.

11. Chen X, Horiuchi A, Kikuchi N, et al. Hedgehog signal pathway is activated in ovarian carcinomas, correlating with cell proliferation: it's inhibition leads to growth suppression and apoptosis. Cancer Sci. 2007; 98: 68-76.

12. Teglund S,Toftgard R. Hedgehog beyond medulloblastoma and basal cell carcinoma. Biochim Biophys Acta. 2010; 1805: 181-208.

13. Ingham P W, McMahon A P. Hedgehog signaling in animal development: paradigms and principles. Genes Dev. 2001; 15: 3059-87.

14. Bhattacharya R, Kwon J, Ali B, et al. Role of hedgehog signaling in ovarian cancer. Clin Cancer Res. 2008; 14: 7659-66.

15. Qualtrough D, Buda A, Gaffield W, et al. Hedgehog signalling in colorectal tumour cells: induction of apoptosis with cyclopamine treatment. Int J Cancer. 2004; 110: 831-7.

16. Kayed H, Kleeff J, Keleg S, et al. Indian hedgehog signaling pathway: expression and regulation in pancreatic cancer. Int J Cancer. 2004; 110: 668-76.

17. Huang S, He J, Zhang X, et al. Activation of the hedgehog pathway in human hepatocellular carcinomas. Carcinogenesis. 2006; 27: 1334-40.

18. Xie Y, Lu W, Liu S, et al. MMP7 interacts with ARF in nucleus to potentiate tumor microenvironments for prostate cancer progression in vivo. Oncotarget. 2016; 7: 47609-47619

19. Wang D, Hu G, Du Y, et al. Aberrant activation of hedgehog signaling promotes cell proliferation via the transcriptional activation of forkhead Box M1 in colorectal cancer cells. J Exp Clin Cancer Res. 2017; 36: 23.

20. Chen $Q, X u R, Z$ eng $C$, et al. Down-regulation of Gli transcription factor leads to the inhibition of migration and invasion of ovarian cancer cells via integrin beta4-mediated FAK signaling. PLoS One. 2014; 9: 5148-52.

21. Lauth M, Bergstrom A, Shimokawa T, et al. Inhibition of GLI-mediated transcription and tumor cell growth by small-molecule antagonists. Proc Natl Acad Sci U S A. 2007; 104: 8455-60.

22. Gross J, Lapiere C M. Collagenolytic activity in amphibian tissues: a tissue culture assay. Proc Natl Acad Sci U S A. 1962; 48: 1014-22.

23. Kessenbrock K, Plaks V, Werb Z. Matrix metalloproteinases: regulators of the tumor microenvironment. Cell. 2010; 141: 52-67.

24. Yokoyama Y, Grunebach F, Schmidt S M, et al. Matrilysin (MMP-7) is a novel broadly expressed tumor antigen recognized by antigen-specific T cells. Clin Cancer Res. 2008; 14: 5503-11.

25. Chang M C, Chen C A, Chen P J, et al. Mesothelin enhances invasion of ovarian cancer by inducing MMP-7 through MAPK/ERK and JNK pathways. Biochem J. 2012; 442: 293-302.

26. Wang F Q, So J, Reierstad S, et al. Matrilysin (MMP-7) promotes invasion of ovarian cancer cells by activation of progelatinase. Int J Cancer. 2005; 114: 19-31.

27. Zhao H, Yang Z, Wang $X$, et al. Triptolide inhibits ovarian cancer cell invasion by repression of matrix metalloproteinase 7 and 19 and upregulation of E-cadherin. Exp Mol Med. 2012; 44: 633-41.

28. Luo S, Zhang B, Dong X P, et al. HSP90 beta regulates rapsyn turnover and subsequent AChR cluster formation and maintenance. Neuron. 2008; 60: 97-110.

29. Luo S W, Zhang C, Zhang B, et al. Regulation of heterochromatin remodelling and myogenin expression during muscle differentiation by FAK interaction with MBD2. Embo j. 2009; 28: 2568-82.

30. Hall T M, Porter J A, Beachy P A, et al. A potential catalytic site revealed by the 1.7-A crystal structure of the amino-terminal signalling domain of Sonic hedgehog. Nature. 1995; 378: 212-6.

31. Yan R, Peng X, Yuan X, et al. Suppression of growth and migration by blocking the Hedgehog signaling pathway in gastric cancer cells. Cell Oncol (Dordr). 2013; 36: 421-35.

32. Liang C C, Park A Y, Guan J L. In vitro scratch assay: a convenient and inexpensive method for analysis of cell migration in vitro. Nat Protoc. 2007; 2: 329-33. 
33. Shi C, Huang D, Lu N, et al. Aberrantly activated Gli2-KIF20A axis is crucial for growth of hepatocellular carcinoma and predicts poor prognosis. Oncotarget. 2016; 7: 26206-19.

34. Tao Y, Shen C, Luo S, et al. Role of Erbin in ErbB2-dependent breast tumor growth. Proc Natl Acad Sci U S A. 2014; 111: E4429-38.

35. Gyorffy B, Lanczky A, Szallasi Z. Implementing an online tool for genome-wide validation of survival-associated biomarkers in ovarian-cancer using microarray data from 1287 patients. Endocr Relat Cancer. 2012; 19: 197-208.

36. Coenraads P J, Van Der Walle H, Thestrup-Pedersen K, et al. Construction and validation of a photographic guide for assessing severity of chronic hand dermatitis. Br J Dermatol. 2005; 152: 296-301.

37. Yamamoto $\mathrm{H}$, Itoh $\mathrm{F}$, Adachi $\mathrm{Y}$, et al. Relation of enhanced secretion of active matrix metalloproteinases with tumor spread in human hepatocellular carcinoma. Gastroenterology. 1997; 112: 1290-6.

38. Fukuda A, Wang S C, Morris J P t, et al. Stat3 and MMP7 contribute to pancreatic ductal adenocarcinoma initiation and progression. Cancer Cell. 2011; 19: 441-55.

39. Hadler-Olsen E, Winberg J O, Uhlin-Hansen L. Matrix metalloproteinases in cancer: their value as diagnostic and prognostic markers and therapeutic targets. Tumour Biol. 2013; 34: 2041-51.

40. Nusslein-Volhard C,Wieschaus E. Mutations affecting segment number and polarity in Drosophila. Nature. 1980; 287: 795-801.

41. Hui C C,Angers S. Gli proteins in development and disease. Annu Rev Cell Dev Biol. 2011; 27: 513-37.

42. Xie J, Murone M, Luoh S M, et al. Activating Smoothened mutations in sporadic basal-cell carcinoma. Nature. 1998; 391: 90-2.

43. Sanchez P, Hernandez A M, Stecca B, et al. Inhibition of prostate cancer proliferation by interference with SONIC HEDGEHOG-GLI1 signaling. Proc Natl Acad Sci U S A. 2004; 101: 12561-6.

44. Feldmann G, Dhara S, Fendrich V, et al. Blockade of hedgehog signaling inhibits pancreatic cancer invasion and metastases: a new paradigm for combination therapy in solid cancers. Cancer Res. 2007; 67: 2187-96.

45. Berman D M, Karhadkar S S, Maitra A, et al. Widespread requirement for Hedgehog ligand stimulation in growth of digestive tract tumours. Nature. 2003; 425: 846-51.

46. Beachy P A, Karhadkar S S, Berman D M. Tissue repair and stem cell renewal in carcinogenesis. Nature. 2004; 432: 324-31.

47. Park H L, Bai C, Platt K A, et al. Mouse Gli1 mutants are viable but have defects in SHH signaling in combination with a Gli2 mutation. Development. 2000; 127: 1593-605.

48. Chaffer C L,Weinberg R A. A perspective on cancer cell metastasis. Science. 2011; 331: 1559-64.

49. Coussens L M, Fingleton B, Matrisian L M. Matrix metalloproteinase inhibitors and cancer: trials and tribulations. Science. 2002; 295: 2387-92

50. Bergers G, Brekken R, McMahon G, et al. Matrix metalloproteinase- 9 triggers the angiogenic switch during carcinogenesis. Nat Cell Biol. 2000; 2: 737-44.

51. Sawada K, Mitra A K, Radjabi A R, et al. Loss of E-cadherin promotes ovarian cancer metastasis via alpha 5-integrin, which is a therapeutic target. Cancer Res. 2008; 68: 2329-39.

52. Sakata K, Shigemasa K, Uebaba $\mathrm{Y}$, et al. Expression of matrix metalloproteinases- 2 and -9 by cells isolated from the peritoneal fluid of women with ovarian carcinoma. Acta Cytol. 2002; 46: 697-703.

53. Byers L J, Osborne J L, Carson L F, et al. Increased levels of laminin in ascitic fluid of patients with ovarian cancer. Cancer Lett. 1995; 88: 67-72.

54. Song N, Liu H, Ma X, et al. Placental growth factor promotes metastases of ovarian cancer through MiR-543-regulated MMP7. Cell Physiol Biochem. 2015; 37: 1104-12.

55. Schmid S, Bieber M, Zhang F, et al. Wnt and hedgehog gene pathway expression in serous ovarian cancer. Int J Gynecol Cancer. 2011; 21: 975-80.

56. Steg A, Wang $\mathrm{W}$, Blanquicett $\mathrm{C}$, et al. Multiple gene expression analyses in paraffin-embedded tissues by TaqMan low-density array: Application to hedgehog and Wnt pathway analysis in ovarian endometrioid adenocarcinoma. J Mol Diagn. 2006; 8: 76-83.

57. Ke Z, Caiping S, Oing Z, et al. Sonic hedgehog-Gli1 signals promote epithelial-mesenchymal transition in ovarian cancer by mediating PI3K/AKT pathway. Med Oncol. 2015; 32: 368.

58. Ciucci A, De Stefano I, Vellone V G, et al. Expression of the glioma-associated oncogene homolog 1 (gli1) in advanced serous ovarian cancer is associated with unfavorable overall survival. PLoS One. 2013; 8: e60145.

59. Schummer M, Drescher C, Forrest R, et al. Evaluation of ovarian cancer remission markers HE4, MMP7 and Mesothelin by comparison to the established marker CA125. Gynecol Oncol. 2012; 125: 65-9. 